\subsection{THE GLOBAL BURDEN OF DISEASE 2010: ESTIMATING BURDENS AT ATTRIBUTABLE TO NUTRITIONAL AND METABOLIC RISK FACTORS: METHODS AND FINDINGS}

\author{
Chair: Dr. John Powles, UK \\ 05-3.1 GLOBAL, REGIONAL AND NATIONAL TRENDS IN \\ METABOLIC RISK FACTORS OF CHRONIC DISEASES: \\ ANALYSIS OF HEALTH SURVEYS AND EPIDEMIOLOGIC \\ STUDIES SINCE 1980
}

doi:10.1136/jech.2011.142976b.49

\begin{abstract}
${ }^{1} \mathrm{G}$ Danaei, ${ }^{*}{ }^{2} \mathrm{G}$ Stevens, ${ }^{1} \mathrm{M}$ Finucane, ${ }^{1} \mathrm{~J}$ Lin, ${ }^{1} \mathrm{G}$ Singh, ${ }^{1,3} \mathrm{C}$ Paciorek, ${ }^{2} \mathrm{M}$ Cowan, ${ }^{1}$ F Farzadfar, ${ }^{4} \mathrm{~S}$ Lim, ${ }^{2} \mathrm{~L}$ Riley, ${ }^{2} \mathrm{H}$ Gutierrez, ${ }^{2} \mathrm{Y}$ Lu, ${ }^{2} \mathrm{~A}$ Bahalim, ${ }^{5} \mathrm{P}$ Pelizzari, ${ }^{1,6} \mathrm{M}$ Ezzati. ${ }^{1}$ Harvard School of Public Health, Boston, Massachusetts, USA; ${ }^{2}$ World Health Organization, Geneva, Switzerland; ${ }^{3}$ University of California, Berkeley, California, USA; ${ }^{4}$ Institute for Health Metrics and Evaluation, Seattle, Washington, USA; ${ }^{5}$ Johns Hopkins Bloomberg School of Public Health, Baltimore, Maryland, USA; ' $I m p e r i a l$ College, London, UK
\end{abstract}

Introduction Information on levels and trends of risk factors is essential for policy making. However, very few comprehensive and consistent global estimates are available. We collected a global database of population level summaries of systolic blood pressure (SBP), body mass index (BMI), serum total cholesterol (TC) and fasting plasma glucose (FPG) and estimated levels and trends since 1980.

Methods For each risk factor, we searched for published articles, analysed health surveys, and included unpublished reports. We excluded non-random samples or self-reported measurements. We used a Bayesian hierarchical model with a non-linear age component and a smoothing time trend for each sex separately.

Results BMI had the most available data (960 country-years), followed by SBP (786), FPG (346) and TC (321). SBP declined in high-income regions by 2.1 to $3.9 \mathrm{~mm} \mathrm{Hg} /$ decade, remained the same in East and South Asia and increased in Oceania and Eastern sub-Saharan Africa. TC declined in high-income regions by $0.2 \mathrm{mmol} / \mathrm{l} /$ decade and increased in East and South-East Asia and Pacific by $0.08-0.09 \mathrm{mmol} / \mathrm{l} /$ decade. BMI increased in virtually all regions with a global slope of $0.4 \mathrm{~kg} / \mathrm{m}^{2} /$ decade in men and 0.5 in women. FPG levels rose by $0.07-0.08 \mathrm{mmol} / \mathrm{l} /$ decade globally. FPG rose in all regions except for East and South-East Asia and Central and Eastern Europe.

Conclusion There was a large variation in levels and trends of metabolic risk factors. Population and individual-level interventions should be formulated to continue beneficial trends and reverse the hazardous ones. National health surveys are essential in monitoring such interventions.

\section{5-3.2 CHARACTERISING GLOBAL DIETARY HABITS RELATED TO CHRONIC DISEASE IN THE 21ST CENTURY: CURRENT KNOWLEDGE AND REMAINING GAPS}

doi:10.1136/jech.2011.142976b.50

${ }^{1} \mathrm{R}$ Micha, ${ }^{*} \mathrm{~S}$ Khatibzadeh, ${ }^{1,2} \mathrm{M}$ Ezzati, ${ }^{1,3} \mathrm{D}$ Mozaffarian. ${ }^{1}$ Harvard School of Public Health, Boston, Massachusetts, USA; ${ }^{2}$ Imperial College London, London, UK; ${ }^{3}$ Harvard Medical School, Boston, Massachusetts, USA

Introduction Understanding the impact of diet on chronic diseases globally has been limited by lack of systematically collected and comparable data on dietary habits on a global scale.

Methods We developed methods to collect nationally representative data on exposure distributions of 20 major dietary risk factors globally and in 21 world regions (196 countries), including total energy, fruits, fruit juices, vegetables, beans / legumes, nuts / seeds, whole-grains, red meats, processed meats, milk, sugar-sweetened beverages, saturated, polyunsaturated, seafood omega-3, plant omega-3, and trans fats, and dietary fibre, sodium, cholesterol and calcium. From Mar/2008 to Oct/2010, searches were performed using multiple online databases, hand-searching of references, and direct author contact. Standardised protocols for quality assessment, data analysis and data extraction were developed.

Results Relevant published data were very limited, requiring direct author contact ( $85 \%$ of the surveys). We identified and obtained data from 164 nationally and 21 non-nationally representative surveys from 79 countries in 17 regions. Four regions of the world lacked representative data: Latin America-Andean, and East, Central, and West Sub-Saharan Africa. Survey sample sizes ranged from $>10000$ (34 surveys), 5000-10000 (23), 1000-5000 (90), and $<1000$ (38). Dietary assessment methods included FFQs (86), diet recall/records (88), and others (11). Data were most frequently available for fruits, vegetables, red meats, and milk, and least for trans-fats, plant omega-3, and polyunsaturated fats.

Conclusion A systematic, comprehensive, and global search allowed compilation of dietary data for most world regions, providing data for estimation of global impact on chronic diseases. Insufficient data in certain regions and for specific nutrients is also evident.

\section{5-3.3 GLOBAL BURDENS OF CHRONIC DISEASE ATTRIBUTABLE TO SUBOPTIMAL DIETARY HABITS: CHALLENGES AND ADVANCES}

doi:10.1136/jech.2011.142976b.51

${ }^{1} \mathrm{R}$ Micha, ${ }^{1} \mathrm{G}$ Danaei, ${ }^{2} \mathrm{~J}$ Powles, ${ }^{1,3} \mathrm{M}$ Ezzati, ${ }^{1,4} \mathrm{D}$ Mozaffarian. ${ }^{*}{ }^{1}$ Harvard School of Public Health, Boston, Massachusetts, USA; ${ }^{2}$ University of Cambridge, Cambridge, UK ${ }^{3}$ Imperial College London, London, UK; ${ }^{4}$ Harvard Medical School, Boston, Massachusetts, USA

Introduction Little quantitative data are available on the global impact of diet on chronic diseases, including cardiovascular diseases, type 2 diabetes and cancer.

Methods We used a comparative risk assessment framework to develop systematic and comparable methods to establish for selected dietary risk factors the effect sizes of probable or convincing causal diet-disease relationships; the alternative minimum risk exposure distribution; and the exposure distribution. These inputs, together with disease-specific mortality rates, allow computation of the numbers of events attributable to each dietary factor globally and in 21 world regions.

Results Using WHO evidence criteria for convincing / probable causal effects, we identified potential causal diet-disease relationships. Effect sizes and ranges of uncertainty were derived from published or de-novo systematic reviews and meta-analyses of trials or high-quality observational studies. Alternative minimum-risk distributions were identified based on amounts corresponding to lowest disease rates in existing populations. Optimal and alternative definitions for each diet exposure were established based on the data used to quantify the harmful or protective effects. We developed methods for identifying and obtaining comparable data from nationally representative diet surveys throughout the world (see separately submitted Abstract). Multi-level hierarchical models will be developed and will be presented to handle the diversity of diet metrics, units of measure, energy-adjustment techniques, and to impute missing data.

Conclusions These new methods will allow, for the first time, comparable and quantitative assessment of the global impact of specific dietary factors on chronic disease mortality. Such global assessment is not only possible, but imperative for priority setting and policy making. 